\title{
Formes familiales des cancers colorectaux
}

\author{
J.-C. Saurin \\ (C) Springer-Verlag France 2011
}

Dans le cadre des prédispositions génétiques au cancer colorectal, le gastroentérologue est le point de convergence des informations génétiques, biologiques et cliniques, dont la complexité a augmenté du fait simplement des progrès considérables qui ont été réalisés dans ces différents domaines. Le gastroentérologue reste et doit rester le praticien de référence pour la prise en charge clinique des polyposes digestives et de la maladie de Lynch, en collaboration étroite avec les oncogénéticiens.

Cette position a des implications en termes de responsabilité. Cela implique la connaissance des algorithmes de prise en charge de ces patients et de leur actualisation régulière. Dans ce but, ce dossier thématique apporte la mise à jour des prises en charge cliniques de ces principaux syndromes, du plus fréquent qu'est la maladie de Lynch, aux plus rares que sont les polyposes hamartomateuses, en passant par les classiques polyposes adénomateuses y compris la dernière-née, la polypose liée au gène $M u t Y H$, dont la prise en charge après diagnostic est finalement assez proche de celle liée au gène $A P C$. En plus de ce cahier thématique, rappelons l'existence de fiches simplifiées de prise en charge éditées par un groupe de travail national (le Genmad) et disponibles sur le site Internet des sociétés savantes.

Cela implique aussi la connaissance et la compréhension des tests génétiques et biologiques, ce qui reste assez simple dans le cadre des polyposes (mutation présente ou absente) mais devient plus complexe dans le cadre des tests moléculaires sur tumeur qui font de plus en plus partie de notre quotidien dans le cadre des suspicions de maladie de Lynch : aux classiques recherches d'instabilité et de perte d'expression d'une protéine par immunohistochimie traduisant le mécanisme de cancérogenèse s'ajoutent aujourd'hui des tests permettant de différencier les cas sporadiques des cas familiaux (mutation de BRAF, surtout test de méthylation du promoteur de MLH1). Or, ces tests moléculaires ont une importance capitale aujourd'hui à la fois pour identifier les personnes suspectes de maladie de Lynch et pour orienter la recherche génétique sur un gène spécifique. Reste le groupe

J.-C. Saurin $(\bowtie)$

Service d'hépatogastroentérologie, pavillon H, hôpital E.-Herriot,

5, place d'Arsonval, F-69437 Lyon cedex 03, France

e-mail : jean-christophe.saurin@chu-lyon.fr très important des situations d'agrégations familiales de cancers ou de polyposes sans syndrome génétique identifié, pour lequel une grosse dose de bon sens et des discussions clinicien-généticien sont de mise pour proposer la meilleure prise en charge.

En amont du gastroentérologue, un travail considérable est réalisé par les oncogénéticiens et biologistes moléculaires. L'article de S. Olschwang rappelle les modalités précises, mais surtout l'importance pratique majeure du diagnostic d'une prédisposition : pour cibler la prise en charge, pour appliquer exactement la prise en charge requise, en insistant sur l'impact émotionnel fort de cette consultation et des résultats qui en découlent, en particulier dans le cadre du diagnostic prédictif qui doit systématiquement être réalisé par un oncogénéticien. Une des nouveautés importantes de ces dernières années est l'accès, pour les familles qui le désirent, à un diagnostic prénatal de l'anomalie génétique responsable de la prédisposition. Encore faut-il développer les moyens très limités en France (trois centres) de ce diagnostic dans sa version préimplantatoire souvent mieux vécue par les familles.

Quels sont les limites actuelles et les objectifs d'amélioration pour le futur? Sans doute une forte hétérogénéité des pratiques de prise en charge (endoscopique, chirurgicale) des patients, surtout dans le cadre des polyposes, soulignant l'intérêt de ce type de cahier thématique, mais aussi des efforts nationaux de synthèse simplifiée à destination des praticiens (expertises de l'INCa récente sur la polypose MYH et la chirurgie prophylactique, fiches pratiques du Genmad). Par ailleurs, l'article commun à plusieurs équipes françaises présenté dans ce cahier et consacré aux limites du dépistage génétique montre bien les difficultés présentes qui aboutissent au fait que près de $50 \%$ des familles ayant une maladie de Lynch ne sont pas identifiées en France, en 2011, contre moins de $20 \%$ des familles à très haut risque de cancer mammaire. Les efforts à faire sont identifiés : la pratique systématique des tests moléculaires (instabilité, immunohistochimie) chez les patients présentant un cancer colorectal avant 60 ans, le rappel des patients pour lesquels un tel test a été réalisé mais qui n'ont pas été adressés/n'ont pas voulu aller en consultation de génétique (40 à $66 \%$ des personnes identifiées par les tests moléculaires!), l'interrogatoire systématique des patients... 